\title{
Pitfalls of Commonly Used Ischemic and Dementia Models Due to Early Seizure by Carotid Ligation
}

\author{
Tsukasa HiRAnO, ${ }^{1}$ Takeshi MiKami, ${ }^{1}$ Shoto YAMADA,,${ }^{1}$ Hiroshi NAGAHAMA, ${ }^{2}$ \\ Rei ENATSU, ${ }^{1}$ Satoshi OOKAWA, ${ }^{1}$ Yukinori AKIYAMA, ${ }^{1}$ \\ and Nobuhiro MIKUNI ${ }^{1}$
}

\begin{abstract}
${ }^{1}$ Department of Neurosurgery, Sapporo Medical University, Sapporo, Hokkaido, Japan ${ }^{2}$ Division of Radioisotope Research, Biomedical Research, Education and Instrumentation Center, Sapporo Medical University School of Medicine, Sapporo, Hokkaido, Japan
\end{abstract}

\begin{abstract}
While the bilateral common carotid artery (CCA) ligation model is widely used in cerebrovascular disease and dementia studies, it can frequently cause seizures. We examined the validity of seizure as an experimental model of ischemia. Eight-week-old male Wistar and Sprague-Dawley (SD) rats were implanted with electrocorticography (ECoG) electrodes and bilateral CCA ligation was performed and compared to the sham groups. ECoG monitoring was used to confirm the seizure discharge and count the number of spikes in the interictal phase $2 \mathrm{~h}$ after ligation, followed by power spectral analysis. Magnetic resonance imaging (MRI) was performed $6 \mathrm{~h}$ after bilateral CCA ligation to assess fractional anisotropy (FA), apparent diffusion coefficient (ADC), and cerebral blood flow (CBF) values. Magnetic resonance spectroscopy (MRS) was also performed and the ischemic parameters and electrophysiological changes were compared. The Wistar rat group had significantly higher mortality, frequency of seizures, incidence of non-convulsive seizures, and number of spikes in the interictal period compared to those in the SD rat group. Power spectral analysis showed increased power in the delta band in both Wistar and SD rat groups. MRI, after CCA ligation, showed significantly lower ADC values, lower glutamine and glutamate levels, and higher lactate values in Wistar rats, although there was no difference in FA values. Metabolic and electrophysiological changes after CCA ligation differed according to the rat strain. Wistar rats were prone to increased lactate and decreased glutamine and glutamate levels and the development of status epilepticus. Seizures can affect the results of ischemic experiments.
\end{abstract}

Keywords: seizure, bilateral carotid artery ligation, brain ischemia

\section{Introduction}

Cerebrovascular disease is the most common cause of epilepsy, accounting for 6.5 to $11 \%$ of all cases. ${ }^{1-3)}$ Post-stroke epilepsy is related to early seizure, which is associated with mortality and morbidity. ${ }^{4,5)}$ Moreover, status epilepticus due to stroke has a poor prognosis. ${ }^{6)}$ Therefore, critical management is indispensable for controlling early seizures. Early seizure in the ischemic area might be caused by metabolic

Received October 27, 2020; Accepted December 9, 2020

Copyright $@ 2021$ by The Japan Neurosurgical Society This work is licensed under a Creative Commons AttributionNonCommercial-NoDerivatives International License. stress in the vulnerable tissue. Sudden ATP depletion accelerates anaerobic metabolism and increases intracellular acidosis. ${ }^{7)}$ These trigger depolarization of the presynaptic neuronal membrane and surrounding astrocytes due to the influx of $\mathrm{Na}+$ ions. Hence, ischemia is closely related to seizure occurrence.

Bilateral rat common carotid artery (CCA) occlusion models are currently widely used in experiments on ischemia and dementia. ${ }^{8-11)}$ Although this model is convenient and results in a stable degree of ischemia, it causes a relatively high rate of seizures. Particularly in the critical developmental window (P 6-12), a period of synaptic maturation and ischemia-induced seizure models have been commonly achieved as a neonatal hypoxia-induced seizure model. ${ }^{12)}$ Ischemia is associated with neuronal 
hyperstimulation and early seizure due to global ischemia, although the significance of electroneurophysiology in adult models has not been clearly elucidated. Early epilepsy after global ischemia may affect the prognosis or results of experiments in ischemia or dementia studies. Therefore, the present study focused on epileptogenicity due to ischemia in commonly used ischemic and dementia models and discussed the factors influencing seizure occurrence in ischemic conditions.

\section{Materials and Methods}

\section{Animal model}

Eight-week-old male Wistar and Sprague-Dawley (SD) rats (Charles River, Yokohama, Japan) were studied according to a protocol approved by the Animal Care and Use Committee of Sapporo Medical University. Before and after the surgical intervention, the rats were housed in a sound-attenuated room at $25 \pm 2^{\circ} \mathrm{C}$ under $12 \mathrm{~h}$ light/dark conditions (8.00 am-8.00 pm) with free access to water and food.

In all, 10 Wistar rats and 10 SD rats underwent bilateral CCA ligation, with another 10 Wistar rats exposed to CCA as the SHAM group. These 30 rats were then prepared for magnetic resonance imaging (MRI). Similarly, 20 Wistar rats and 10 SD rats were prepared for the electrocorticography (ECoG) study. Of these, 10 Wistar rats and 10 SD rats underwent bilateral CCA ligation, while another 10 Wistar rats were exposed to bilateral CCA as the SHAM group. Bilateral CCA ligation was performed as follows. The rats were anesthetized with an intraperitoneal injection of ketamine $(75 \mathrm{mg} / \mathrm{kg}$ ) and xylazine $(10 \mathrm{mg} / \mathrm{kg})$. After ventral neck incision, both CCAs were exposed from the surrounding tissue while preserving the vagus nerve and omohyoid muscle. Both CCAs were then permanently occluded with 4-0 silk.

\section{ECoG recording and analysis}

Anesthesia and analgesia were performed similarly. The animals were placed in the abdominal position. After making a mid-sagittal incision, two pilot holes were placed on the skull bilaterally $2 \mathrm{~mm}$ ventral and $3 \mathrm{~mm}$ lateral from the bregma. Two additional pilot holes were placed bilaterally $3 \mathrm{~mm}$ dorsal and $3 \mathrm{~mm}$ lateral from the bregma. An ECoG recording system (Pinnacle Technology, Inc., Lawrence, KS, USA) was used for detection and analysis. The ECoG electrodes penetrated the dura mater and were fixed to the skull. Electromyograms were recorded from two wire electrodes implanted in the subfascial layer of the nuchal muscle. ECoG and EMG were amplified (10×), high-pass filtered $(0.5 \mathrm{~Hz}$ for ECoG and $10 \mathrm{~Hz}$ for EMG), and low-pass filtered $(30 \mathrm{~Hz}$
ECoG and $40 \mathrm{~Hz}$ EMG). The signals were then sampled at $400 \mathrm{~Hz}$ and digitized using a 14-bit A/D converter. The ECoG and EMG signals were continuously recorded for $24 \mathrm{~h}$.

During the ECoG recording, the rats were allowed to move freely in their cages by means of a rotating electrical connector. The ECoG and video monitoring were recorded for $24 \mathrm{~h}$ after both CCAs were occluded. The number of convulsive seizures and subclinical seizures were counted. The convulsive seizures were defined based on a modified Racine scale, ${ }^{13,14)}$ as described previously: (1) mouth and facial movement, (2) head nodding, (3) unilateral forelimb clonus, (4) bilateral forelimb clonus, (5) rearing and falling, and (6) wild running and jumping with vocalization. A subclinical seizure was defined as a unilateral or bilateral rhythmic activity on ECoG for more than 10 seconds without seizures. ${ }^{15,16)}$

Two hours after bilateral CCA ligation, two examiners counted the number of spikes observed in 10 min and calculated the average number of spikes from the left and right electrodes. A spike was defined as a burst wave with an amplitude exceeding $40 \mu \mathrm{V}$, independent of background activity. The spectral power ratio was then calculated by a fast Fourier transform over the range of $0.5 .30 \mathrm{~Hz}$ using SIRENIA SEIZURE PRO (Pinnacle Technology, Inc.). The number of points in the fast Fourier transform was set to 500. The frequency range of $0.5 .30 \mathrm{~Hz}$ was split into the delta $(0.5 .4 \mathrm{~Hz})$, theta $(4-8 \mathrm{~Hz})$, alpha (8-14 Hz), and beta (14-30 Hz) frequency bands. To compare the spectral power of each band, the spectral power of each band to that of the whole was calculated as follows: $\mathrm{E} / \mathrm{DTAB}=$ (spectral power of each band)/(sum of the spectral powers of the four bands), where $\mathrm{E}$ is the spectral power of the delta, theta, alpha, or beta band.

\section{MRI assessment and analysis}

Imaging was performed before occlusion as a control and $6 \mathrm{~h}$ after occlusion. The rats were anesthetized using an isoflurane/oxygen mixture ( $5 \%$ for induction and $2 \%$ for maintenance) and fitted with a custom-made nose cone to deliver inhalant anesthetics. MRI was performed using a 7.0 T magnet (Bruker, Pharma Scan, Germany). Radiofrequency transmission and reception were accomplished with a $40 \mathrm{~mm}$ inner diameter volume coil for brain imaging. Diffusion tensor imaging (DTI) was performed with single-shot, spin-echo echoplanar images (EPI) acquired with a $128 \times 80$ matrix (reconstructed to $128 \times 128$ matrix), $30.0 \times$ $30.0 \mathrm{~mm}^{2}$ field of view (FOV), $1.0 \mathrm{~mm}$ control slices, repetition time (TR) $=3 \mathrm{~s}, \mathrm{~b}=1000 \mathrm{sec} / \mathrm{mm}^{2}$, echo time $(\mathrm{TE})=26.36 \mathrm{~ms}$, gradient directions $=128$, 
$\Delta=8.5 \mathrm{~ms}, \delta=2.5 \mathrm{~ms}$, and 1 average (total acquisition time $6.39 \mathrm{~min}$ ). Noninvasive quantitative cerebral blood flow (CBF) measurements were performed using the continuous arterial spin-labeling (ASL) technique. Paired images were acquired alternately, one with ASL and the other without spinlabeling preparation (control). Single-shot, spin-echo EPI was acquired with identical parameters except for $\mathrm{TE}=32 \mathrm{~ms}$, TR $=10.2 \mathrm{sec}, 96 \times 96$ matrix, $25 \times 25$ FOV, $2.0 \mathrm{~mm}$ single coronal slice (bregma $0.0 \mathrm{~mm}$ ), $10 \mathrm{~s}$ labeling duration, $200 \mathrm{~ms}$ post-labeling delay, $40 \mathrm{mT} / \mathrm{m}$ labeling gradient, and 8 signal averages (total acquisition time of $2.4 \mathrm{~min}$ ). The labeling slice was positioned $0.0 \mathrm{~mm}$ proximal from the center of the imaging slice.

DICOM images of the DTI data were loaded into the Diffusion Toolkit (version 0.6.4.1) to generate tensor data and scalar maps for tract reconstruction with TrackVis (version 0.6.1). CBF maps obtained from ASL were generated using MATLAB (Mathworks, Natick, MA, USA). Based on the obtained images, each parameter was quantified using ImageJ (1.52a; Java 1.8.0). Regions of interest (ROIs) in the bilateral dorsal cortex and striatum were manually drawn on the fractional anisotropy (FA) and CBF maps in slices at the position of the bregma from each animal and the average CBF, apparent diffusion coefficient (ADC), and FA values were calculated for each ROI. The eigenvalues $(\lambda 1, \lambda 2$, and $\lambda 3)$ of the diffusion tensor were created using the Diffusion Toolkit and used to calculate the ADC and FA. The ratios of the pre-ligation and post-ligation values for each parameter were compared. Hyper-intensity areas (HIAs) in the slice on diffusion-weighted imaging (DWI) were measured by manually subtracting the ROI using OsiriX Lite (ver. 11.0.4, Antonie Rosset, UCLA, Los Angeles, CA, USA). HIA ratios (HIAs/total area) on DWI were compared.

\section{Magnetic resonance spectroscopy analysis}

Imaging was performed before occlusion as a control and $6 \mathrm{~h}$ after occlusion. Initial T1-weighted localizer and T2-weighted imaging (TR: $3000 \mathrm{~ms}$, TE: $30 \mathrm{~ms}$ ) were used to localize the voxel area. A single-voxel point-resolved spectroscopy pulse sequence (TR/TE 2000/30 ms, BW $3301 \mathrm{~Hz}, 2048$ points, 1024 excitations) was used to obtain the spectra. The voxel size was $2 \times 2 \times 3 \mathrm{~mm}$. Spectra were taken from the left striatum before occlusion. Spectra $6 \mathrm{~h}$ after occlusion were taken from the one with more ischemic changes in the left or right striatum, as confirmed by DWI and T2-weighted imaging. A preceding chemical shift-selective radiofrequency pulse suppressed the water proton signal. The magnetic resonance spectroscopy (MRS) sequence duration was $34 \min 8$ seconds. Lactate (Lac), $\mathrm{N}$-acetyl aspartate (NAA), glutamate and glutamine $(\mathrm{Glu}+\mathrm{Gln})$, and total creatine $(\mathrm{tCr}=\mathrm{Cr}+\mathrm{PCr})$ were quantified for each spectrum using LCModel. Each metabolite-to-tCr ratio was assessed. The ratios of the pre-ligation and post-ligation values for each parameter were compared.

\section{Statistical analysis}

Data are expressed as median (interquartile range). All statistical analyses were performed using IBM SPSS Statistics for Windows, version 26.0 (IBM Corp., Armonk, NY, USA). The mortality rates and numbers of convulsive and non-convulsive seizures were analyzed by $\chi^{2}$ tests. The number of spikes, spectral power, FA value, ADC value CBF, and HIA ratio on DWI were analyzed using Kruskal-Wallis tests, followed by Dunn's multiple comparison tests. Significant differences were defined as $p$ values $<0.05$.

\section{Results}

\section{ECoG and video monitoring}

Table 1 shows the presence of seizures, mortality, and electrophysiological findings on ECoG monitoring. The total mortality in the ECoG and MRI studies was $80.0 \%(16 / 20)$ in the Wistar group, $10.0 \%(2 / 20)$ in the SD group, and $0.0 \%(0 / 20)$ in the SHAM group, with significant differences between these groups ( $p<0.001$ ). Video 1 (available online) presents Wistar rats during the interictal and ictal states.

In terms of ECoG findings, convulsive seizures were observed in $60.0 \%(6 / 10)$ of rats in the Wistar group, $20.0 \%(2 / 10)$ in the $S D$ group, and $0.0 \%$ $(0 / 10)$ in the SHAM group, with significant differences between groups $(p<0.001)$. The number of rats with convulsive seizures in the Wistar group was significantly higher $(p<0.01)$ and that in the SHAM group was significantly lower than that in the other groups $(p<0.05)$ in residual analysis. Subclinical seizures were observed in $100.0 \%(10 / 10)$ of rats in the Wistar group (Fig. 1), 40.0\% (4/10) in the SD group, and $0.0 \%(0 / 10)$ in the SHAM group, with significant differences between these groups $(p<0.001)$. The Wistar group showed a significantly higher $(p<0.01)$ and the SHAM group had a significantly lower rate of subclinical seizures than those in the other groups $(p<0.01)$ in residual analysis. During the interictal period at $2 \mathrm{~h}$ after ligation, the Wistar rat group had an average of 20.6 (10.0.26.1) spikes in $10 \mathrm{~min}$, compared to 2.1 (1.7.8.3) in the SD group and $2.5(1.2-3.2)$ in the SHAM group. The number of spikes was significantly higher in the Wistar group than in the SD group $(p=0.01)$. 
Table 1 Electrophysiological findings on ECoG monitoring

\begin{tabular}{lcccccc}
\hline & Wistar & SD & SHAM & $p$ & Wistar-SHAM & Wistar-SD \\
\hline Mortality after $24 \mathrm{~h}$ & $16 / 20(80 \%)$ & $2 / 20(10 \%)$ & $0 / 20(0 \%)$ & $<0.001$ & $<0.001$ & $<0.001$ \\
Subclinical seizure & $10 / 10(100 \%)$ & $4 / 10(40 \%)$ & $0 / 10(0 \%)$ & $<0.001$ & $<0.001$ & 0.011 \\
Convulsive seizure & $6 / 10(60 \%)$ & $2 / 10(20 \%)$ & $0 / 10(0 \%)$ & 0.008 & 0.011 & 0.17 \\
Modified Racine & $1 / 5$ & $1 / 1$ & $0 / 0$ & - & - & 0.001 \\
Scale (5)/(6) & & & & $<-0.001$ & 0.01 \\
Number of spikes & $20.6(10.0-26.1)$ & $2.1(1.7-8.3)$ & $2.5(1.2-3.2)$ & $<0.001$ & 0 \\
\hline
\end{tabular}

ECoG: electrocorticography.

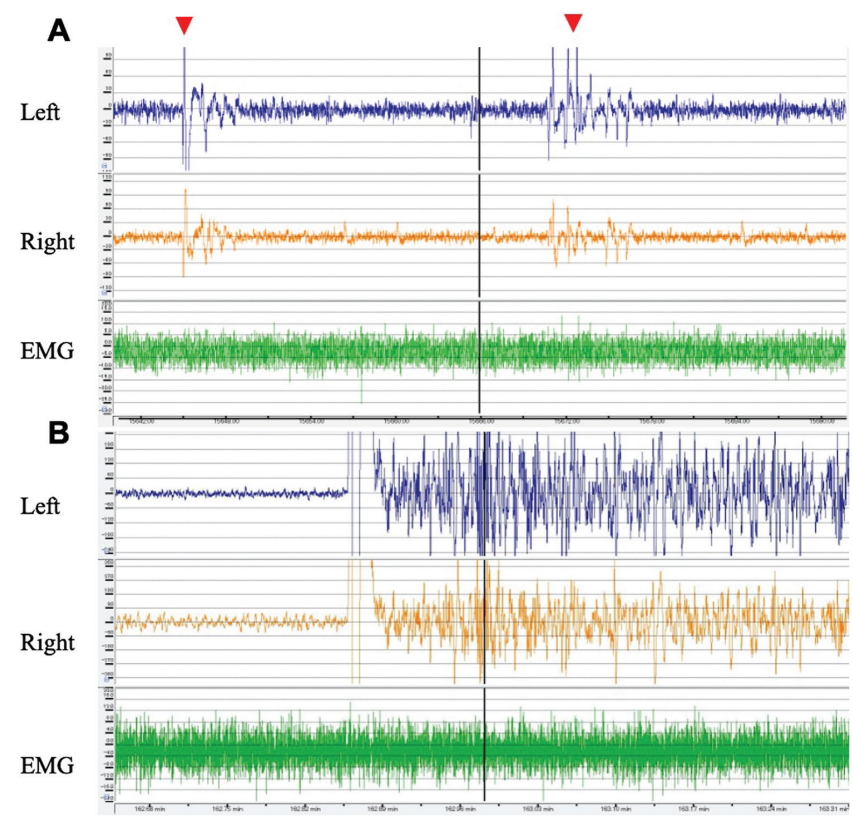

Fig. 1 Epileptiform discharges during $24 \mathrm{~h}$ of monitoring. The top and middle ECoG traces were from the left and right hemispheres, respectively. The bottom trace is the EMG recording from the nuchal muscle. (A) ECoG findings during the interictal state. Occasional spikes are noted in the interictal period (arrowhead). (B) ECoG findings during the ictal state. Rhythmic or repetitive waves in the delta to theta bands are noted during the ictal period. Significant differences were defined as $p$ values $<0.05$. ECoG: electrocorticography, EMG: electromyography.

The median values of the spectral power ratios in the Wistar, SD, and SHAM rat groups, respectively, were as follows (Fig. 2): D/DTAB: 0.49 (0.32-0.67), 0.39 (0.31-0.47), and 0.21 (0.19-0.26); T/DTAB: 0.22 (0.14-0.35), 0.25 (0.19-0.29), and 0.35 (0.29-0.44); A/DTAB: 0.11 (0.89-0.18), 0.13 (0.10$0.17)$, and $0.14(0.10-0.19)$; and B/DTAB: 0.11 (0.08-0.15), $0.16(0.14-0.22)$, and 0.24 (0.17-0.31). The mean DAR was 3.62 (1.94-7.66) in the Wistar rat group, $2.59(1.66 .4 .19)$ in the SD group, and
1.68 (1.15.2.09) in the SHAM group. Regarding delta bands, the spectral power ratios in the Wistar and SD groups were significantly higher than those in the SHAM group (both $p<0.001$ ). Regarding the theta and beta bands, the spectral power ratios in the Wistar and SD rat groups were significantly lower than those in the SHAM rat group (theta: $p<0.01$ for both; beta: $p<0.001$ and $p=0.013$ ). Regarding the alpha bands, no significant difference was observed among the groups $(p=0.413)$. After CCA occlusion, the Wistar rats tended to show increased spectral power of the low-frequency band and decreased power in the high-frequency band.

\section{MRI findings}

Table 2 shows the post- and pre- ligation ratios of FA, ADC, CBF, and HIA on DWI in MRI study. In the dorsal cortex, the FA value was 1.029 (0.961-1.179) in the Wistar group, 1.034 (0.907-1.008) in the SD group, and 1.024 (0.907-1.048) in the SHAM group, with no significant differences between groups. The ADC value was 0.648 (0.556-0.804) in the Wistar group, $0.948(0.907-0.976)$ in the SD group, and 0.979 (0.957-1.004) in the SHAM group, with significant differences between groups $(p<0.001)$. The level in the Wistar group was significantly lower than those in the SD $(p=0.001)$ and SHAM $(p<0.001)$ groups. The CBF value was $0.322(0.268-$ $0.395)$ in the Wistar group, $0.342(0.280-0.510)$ in the SD group, and $0.882(0.718-0.955)$ in the SHAM group, with significant differences between groups $(p<0.001)$. The value in the SHAM group was significantly lower than those in the Wistar $(p<0.001)$ and SD $(p<0.001)$ groups.

In the striatum, the FA value was 1.034 (0.9721.143) in the Wistar group, 1.16 (1.035-1.237) in the SD group, and 1.006 (0.879-1.171) in the SHAM group, with no significant differences between groups. The ADC value was $0.643(0.560-0.948)$ in the Wistar group, $0.950(0.922-1.010)$ in the SD group, and 1.003 (0.989-1.033) in the SHAM group, with significant differences between groups $(p<0.001)$. The values 


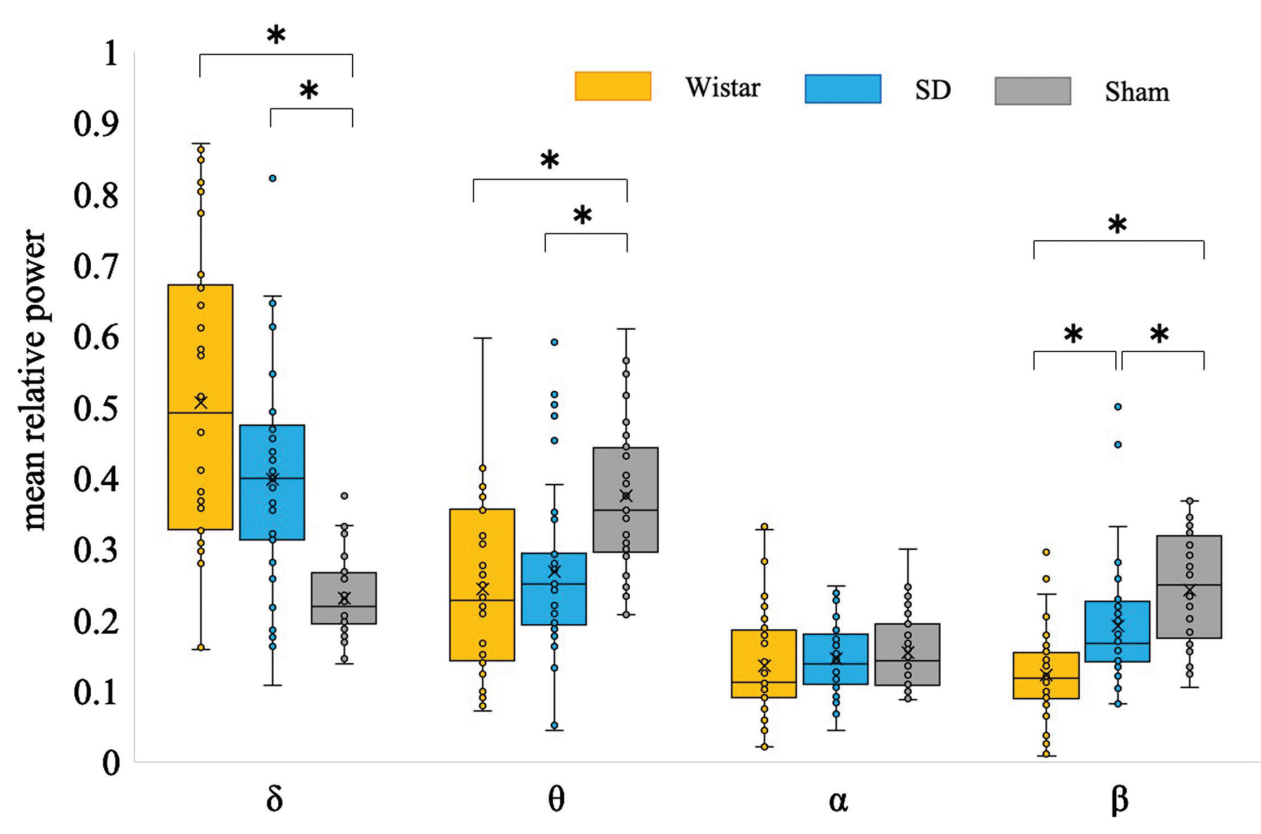

Fig. 2 Comparisons of ECoG spectrum characteristics between the Wistar, SD, and Sham rat groups. The powers in the delta bands of the Wistar and SD groups were significantly higher than those in the Sham group. The powers in the theta bands were significantly lower in both groups than in the Sham group. The power in the beta band was significantly lower in the Wistar group than in the SD group. Significant differences were defined as $p$ values $<0.05$. ECoG: electrocorticography, SD: Sprague-Dawley.

in the Wistar group were significantly lower than those in the SD $(p<0.001)$ and SHAM $(p=0.030)$ groups. The CBF value was $0.451(0.319-0.513)$ in the Wistar group, $0.403(0.325-0.470)$ in the SD group, and $0.826(0.699-0.947)$ in the SHAM group, with significant differences between groups $(p<0.001)$. The values in the SHAM group were significantly lower than those in the Wistar $(p<0.001)$ and SD $(p<0.001)$ groups. The ADC values were significantly decreased in Wistar rats compared to those in SD rats, despite similar decreases in FA and CBF values in both the dorsal cortex and striatum.

The HIA ratio on DWI was $0.371(0.306-0.422)$ in the Wistar group, $0.000(0.000-0.000)$ in the SD group, and $0.000(0.000-0.000)$ in the SHAM group.

\section{MRS findings}

The mean ratio of pre- and post-ligation Glu+Gln value was $0.624(0.595-0.648)$ in the Wistar group, $0.915(0.799-0.965)$ in the SD group, and 1.004 (0.851-1.060) in the SHAM group, with significant differences between groups ( $p=0.001$ ) (Fig. 3). The values in the Wistar group were significantly lower than those in the SD $(p=0.02)$ and SHAM $(p=0.001)$ groups. The NAA value was 0.668 (0.612-0.712) in the Wistar group, 0.984 (0.721-1.027) in the SD group, and $1.024(0.973-1.080)$ in the SHAM group, with significant differences between groups ( $p=0.001)$. The value in the Wistar group was significantly lower than that in the SHAM group $(p=0.001)$. The Lac levels were 6.862 (5.953-8.279) in the Wistar group, $0.930(0.589-1.935)$ in the SD group, and $1.179(0.668-1.892)$ in the SHAM group, with significant differences between groups $(p<0.001)$. The Wistar group had a significantly higher value than those in the SD $(p=0.001)$ and SHAM ( $p=$ 0.002 ) groups. Thus, Glu+Gln and NAA levels were significantly decreased and the Lac level was significantly increased in Wistar rats after CCA ligation.

\section{Discussion}

The results of this study showed that the bilateral CCA occlusion model, which is commonly used in chronic ischemia or dementia studies, can easily develop high-grade fatal status epilepticus in the acute stage of ischemia. The causes of seizures due to cerebral ischemia can be divided into two types according to the stage of seizure occurrence: acute symptomatic seizures caused by acute ischemic metabolites and epilepsy caused by cells damaged by ischemia in the chronic phase. In acute cerebral ischemia, inadequate oxygenation increases intracellular anaerobic metabolism, resulting in intracellular ATP depletion, increased Lac concentration, and intracellular acidification. ${ }^{17)}$ In our study, MRS 
Table 2 Post- and pre-ligation ratios of FA, ADC, CBF, and HIA on DWI in MRI study

\begin{tabular}{|c|c|c|c|c|c|c|c|}
\hline & & Wistar & SD & SHAM & $p$ & Wistar-SHAM & Wistar-SD \\
\hline Dorsal cortex & CBF ratio & $\begin{array}{c}0.322 \\
(0.268-0.395)\end{array}$ & $\begin{array}{c}0.342 \\
(0.280-0.510)\end{array}$ & $\begin{array}{c}0.882 \\
(0.718-0.955)\end{array}$ & $<0.001$ & $<0.001$ & 0.947 \\
\hline \multirow[t]{2}{*}{ Striatum } & ADC ratio & $\begin{array}{c}0.643 \\
(0.560-0.948)\end{array}$ & $\begin{array}{c}0.950 \\
(0.922-1.010)\end{array}$ & $\begin{array}{c}1.003 \\
(0.989-1.033)\end{array}$ & $<0.001$ & $<0.001$ & 0.03 \\
\hline & CBF ratio & $\begin{array}{c}0.451 \\
(0.319-0.513)\end{array}$ & $\begin{array}{c}0.403 \\
(0.325-0.470)\end{array}$ & $\begin{array}{c}0.826 \\
(0.699-0.947)\end{array}$ & $<0.001$ & $<0.001$ & 1 \\
\hline
\end{tabular}

ADC: apparent diffusion coefficient, CBF: cerebral blood flow, DWI: diffusion-weighted imaging, FA: fractional anisotropy,

HIA: hyper-intensity area, MRI: magnetic resonance imaging.

at $6 \mathrm{~h}$ after bilateral CCA ligation in Wistar rats showed a marked increase in Lac, thus confirming increased anaerobic metabolism.

Originally, the mortality rate of the bilateral CCA ligation model was reported as $40-50 \%$, which was not low. ${ }^{18,19)}$ In this study, the bilateral CCA ligation model in Wistar rats showed a mortality rate of $80 \%$ within $24 \mathrm{~h}$, which was relatively higher than those previously reported. This is attributable to two reasons. First, the sex and age of this model were selected to more strongly reflect the influence of ischemia. Second, unexplained deaths during the acute stage may have been excluded in previous reports. The CCA ligation model was created as an experimental model of chronic cerebral hypoperfusion. Conversely, our bilateral CCA ligation models in Wistar rats were used as models of epilepsy induced by ischemia with stable reproducibility.

In the early phase of ischemic conditions, the most important mechanism after ischemia is glutamate excitotoxicity. ${ }^{20,21)}$ Intracellular acidification promotes the excessive release of glutamate into the intercellular space and causes abnormal neuronal excitation. ${ }^{22,23)}$ Glutamate causes a harmful influx of $\mathrm{Ca}^{2}+$ into the cell, leading to excitatory cell death. ${ }^{22,24)}$ The excitatory synapse of the central nervous system includes three major glutamate receptors: a-amino-3-hydroxy-5-methyl-4-isoxazolepropionic acid (AMPA) receptors, multiple mechanisms of ketamine blockade of N-methyl-D-aspartate receptors, and Kainate receptors. ${ }^{25)}$ AMPA receptors have recently been shown to be associated with epileptogenesis. ${ }^{25,26)}$ Perampanel, a noncompetitive AMPA receptor antagonist widely used as a new oral antiepileptic drug, reportedly reduces acute ischemic stroke volume. ${ }^{27-29)}$ AMPA receptor antagonists may be effective in suppressing seizures associated with acute ischemic stroke; thus, the model in this study is useful for future experiments because of the high probability of acute symptomatic seizures. In the present study, we observed a significant decrease in NAA and Glu+Gln in Wistar rats. During acute cerebral ischemia, excessive excitation due to glutamate overload caused seizures; however, in this study, MRS results showed that the glutamate concentrations were low. As a hypothesis of its cause, under acute cerebral ischemia, glutamate biosynthesis is inhibited because the tricarboxylic acid (TCA) cycle fails to function due to glycogen depletion and hypoxia in the brain. Since MRS detects both intracellular and extracellular glutamate, the total amount of glutamate in the brain was likely decreased after ligation. The results of our study showed decreased NAA, Glu, and Gln and increased Lac levels, which have also been observed in cerebral ischemia. ${ }^{30)}$ In patients with epilepsy, such as Rasmussen's syndrome, hemimegalencephaly, and hippocampal sclerosis, decreased NAA and Glu levels were confirmed by MRS. ${ }^{31-33)}$ The decrease in NAA, which is abundant in normal neurons, represents a decrease in the number of neuronal cells and dysfunction, ${ }^{31,34)}$ and an increase in abnormal cells in the voxel. The decrease in Glu and Gln represents a relative decrease, as they are originally synthesized in normal neurons and glial cells. The results of the present study showed a similar trend to those reported in previous studies, showing that bilateral CCA ligation 

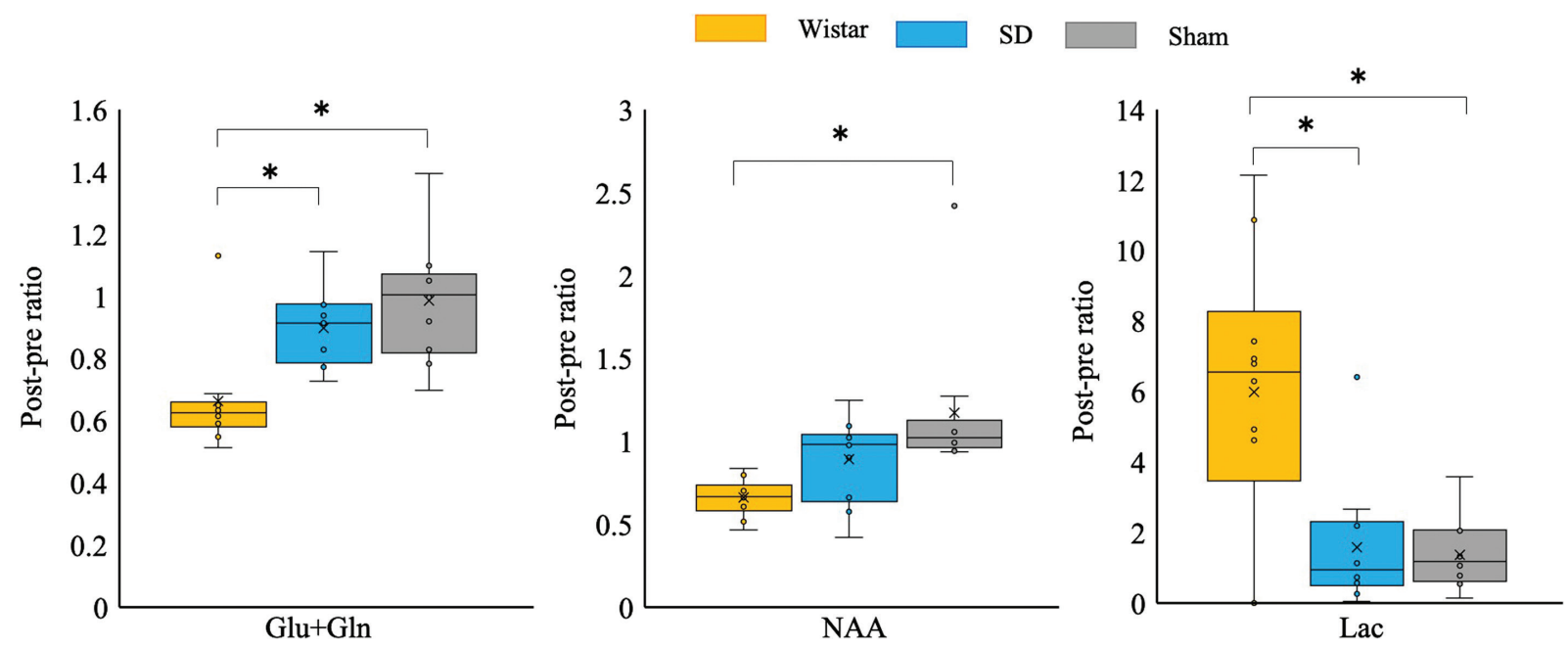

Fig. 3 MRS comparisons of striatum between the Wistar, SD, and Sham rat groups. After bilateral CCA ligation, Glu and Gln levels were significantly decreased in the Wistar group compared to those in the SD and Sham groups. NAA levels were significantly decreased in the Wistar and Sham groups. The Lac level was significantly higher in the Wistar group than in the SD and Sham groups. Significant differences were defined as $p$ values $<0.05$. CCA: common carotid artery, Gln: glutamine, Glu: glutamate, Lac: lactate, MRS: magnetic resonance spectroscopy, NAA: N-acetyl aspartate, SD: Sprague-Dawley.

caused strong cerebral ischemia in Wistar rats. The localization of glutamate in neurons, glial cells, and intercellular spaces, rather than the total amount of glutamate in the brain, leads to seizures during cerebral ischemia. There is a need to histologically assess glutamate localization and receptor activation in future studies.

From the viewpoint of electrophysiology, power spectrum analysis showed an increase in the slowwave component and a decrease in the fast-wave component in the Wistar rat groups. The power of the slow wave, especially in the delta band, is increased during cerebral ischemia. ${ }^{35-38)}$ The increase in spectral power values in the delta band in Wistar rats may reflect the acute ischemic state of the brain. In addition, spectral power analysis of electroencephalograms (EEGs) in epilepsy patients has shown increased power values in slow-wave components such as the delta and theta bands..$^{39,40)}$ In the present study, the increased power values of the slow-wave components in Wistar rats could be explained by the cerebral ischemic state, although this state was prone to concurrent epileptic discharges. Therefore, the bilateral CCA ligation model in Wistar rats has the property of developing status epilepticus caused by acute cerebral ischemia.

Our study results demonstrated that the Wistar rats were more prone to seizures after bilateral CCA ligation compared to SD rats. The reason for this could be a difference in their vulnerabilities to cerebral ischemia. MRI studies after ligation revealed a greater influence of cerebral ischemia in the Wistar group than in the SD group. Although there were no significant differences in CBF and FA levels between the Wistar and SD groups, the ADC levels were lower and the HIA ratio was higher in the Wistar group. The CBF was lower than the control, although the CBF did not differ significantly in the Wistar and SD rats in this study. This is attributable to the setting of the ASL parameter. The labeling duration was set at $10 \mathrm{~s}$ in this study for the insurance of signal-to-noise ratio and repeatability. Thus, the difference may not be observed in the hypoperfusion zone. The Wistar group was more strongly affected by ischemia. The cause of the lower ischemic tolerance in the Wistar rat group may be poor collateral circulation from the posterior circulation. Wistar rats have a smaller posterior communicating artery diameter compared to that of SD rats, in which the differences in white matter damage in the chronic phase between lines have been reported. ${ }^{41)}$ In the present study, the posterior communicating artery was well delineated in the SD rat group but poorly delineated in the Wistar rat group on MRA $6 \mathrm{~h}$ after ligation. Moreover, in a previous study, the CBF in Wistar rats was reduced to nearly $30 \%$ of the pre-ligation level, ${ }^{10,41)}$ similar to the results observed in the present study. Although the Wistar rats did not show any difference in CBF compared to the SD rat group, the anatomical characteristics of the Wistar rats were strongly influenced by ischemia. Another possible reason for the high seizure rate in the Wistar rats is that their neurons may be more vulnerable 
to ischemia compared with SD rats. Consequently, Wistar rats were more vulnerable to ischemia and, therefore, more prone to status epilepticus after bilateral CCA ligation, making it a superior model of status epilepticus after global ischemia.

Regarding the epileptic discharges, the control model also had 2.5 spikes per $10 \mathrm{~min}$, although the stress of the synergistic effects of simultaneous electrode implantation and bilateral CCA ligation may not have influenced mortality. Because the mortality rate of Wistar rats that underwent MRI without electrode implantation was also high, the mortality presented in Table 1 applies to all the rats that underwent MRI or electrode implantation. Moreover, the control group underwent a sham operation to subject the bilateral CCA to CCA occlusion. Thus, surgical stress was minimal in this study.

This study has a limitation. Because of the high mortality rate of Wistar rats during the acute stage, we could not evaluate cognitive function during the chronic phase. Status epilepticus during the acute stage may have caused extensive neuronal damage. The surviving rats also had frequent spikes and subclinical seizures, which suggested cognitive impairment during the chronic phase. Since status epilepticus has been reported to be strongly associated with cognitive function, ${ }^{42-44)}$ we speculate that the impact on cognitive function during the chronic stage may be apparent. These should be examined with adequate agents. Moreover, the bilateral CCA ligation model has been reported to cause atrophy and the deterioration of cognitive function during the chronic phase following hypoperfusion in the cerebral cortex, hippocampus, and basal ganglia. ${ }^{8,10,45)}$ Further studies on the cognitive and histological assessments during the chronic stage are necessary.

\section{Conclusion}

The bilateral CCA ligation model efficiently induced status epilepticus, especially in Wistar rats, as confirmed by electrophysiological studies. Although bilateral CCA ligation has been used as a model of cerebral ischemia and dementia, it is prone to increase Lac and decrease Glu+Gln, thereby inducing seizures. Therefore, the influence of seizures should be considered in experimental studies. Conversely, this model may prove to be a superior model for studies on early seizure after global ischemia.

\section{Acknowledgments}

This work was partially supported by the Japanese Ministry of Education, Culture, Sports, Science, and Technology (20K09352).

\section{Conflicts of Interest Disclosure}

None.

\section{References}

1) Herman ST: Epilepsy after brain insult: targeting epileptogenesis. Neurology 59: S21-26, 2002

2) Hauser WA, Annegers JF, Kurland LT: Incidence of epilepsy and unprovoked seizures in Rochester, Minnesota: 1935-1984. Epilepsia 34: 453-468, 1993

3) Hirano T, Enatsu R, Iihoshi S, et al.: Effects of hemosiderosis on epilepsy following subarachnoid hemorrhage. Neurol Med Chir (Tokyo) 59: 27-32, 2019

4) Burneo JG, Fang J, Saposnik G, Investigators of the Registry of the Canadian Stroke N: Impact of seizures on morbidity and mortality after stroke: a Canadian multi-centre cohort study. Eur J Neurol 17: 52-58, 2010

5) Hesdorffer DC, Benn EK, Cascino GD, Hauser WA: Is a first acute symptomatic seizure epilepsy? Mortality and risk for recurrent seizure. Epilepsia 50: 1102-1108, 2009

6) Tomari S, Tanaka T, Matsubara S, et al.: Risk factors for nonconvulsive status epilepticus after stroke. Eur Neurol 80: 256-260, 2018

7) Abe T, Niizuma K, Kanoke A, et al.: Metabolomic analysis of mouse brain after a transient middle cerebral artery occlusion by mass spectrometry imaging. Neurol Med Chir (Tokyo) 58: 384-392, 2018

8) Farkas E, Luiten PG, Bari F: Permanent, bilateral common carotid artery occlusion in the rat: a model for chronic cerebral hypoperfusion-related neurodegenerative diseases. Brain Res Rev 54: 162-180, 2007

9) Ueno M, Chiba Y, Matsumoto K, et al.: Blood-brain barrier damage in vascular dementia. Neuropathology 36: 115-124, 2016

10) Tsuchiya M, Sako K, Yura S, Yonemasu Y: Cerebral blood flow and histopathological changes following permanent bilateral carotid artery ligation in Wistar rats. Exp Brain Res 89: 87-92, 1992

11) Wakita H, Tomimoto H, Akiguchi I, Kimura J: Glial activation and white matter changes in the rat brain induced by chronic cerebral hypoperfusion: an immunohistochemical study. Acta Neuropathol 87: 484-492, 1994

12) Cuaycong M, Engel M, Weinstein SL, et al.: A novel approach to the study of hypoxia-ischemia-induced clinical and subclinical seizures in the neonatal rat. Dev Neurosci 33: 241-250, 2011

13) Racine RJ: Modification of seizure activity by electrical stimulation. II. Motor seizure. Electroencephalogr Clin Neurophysiol 32: 281-294, 1972

14) Cela E, McFarlan AR, Chung AJ, et al.: An optogenetic kindling model of neocortical epilepsy. Sci Rep 9: 5236, 2019

15) Yoo JY, Rampal N, Petroff OA, Hirsch LJ, Gaspard N: Brief potentially ictal rhythmic discharges in critically ill adults. JAMA Neurol 71: 454-462, 2014 
16) Avdic U, Ahl M, Chugh D, et al.: Nonconvulsive status epilepticus in rats leads to brain pathology. Epilepsia 59: 945-958, 2018

17) Obrenovitch TP, Garofalo O, Harris RJ, et al.: Brain tissue concentrations of ATP, phosphocreatine, lactate, and tissue $\mathrm{pH}$ in relation to reduced cerebral blood flow following experimental acute middle cerebral artery occlusion. J Cereb Blood Flow Metab 8: 866-874, 1988

18) Payan HM, Levine S, Strebel R: Effects of cerebral ischemia in various strains of rats. Proc Soc Exp Biol Med 120: 208-209, 1965

19) Cechetti F, Worm PV, Pereira LO, Siqueira IR, A Netto $\mathrm{C}$ : The modified 2VO ischemia protocol causes cognitive impairment similar to that induced by the standard method, but with a better survival rate. Braz J Med Biol Res 43: 1178-1183, 2010

20) Trinka E, Cock H, Hesdorffer D, et al.: A definition and classification of status epilepticus-Report of the ILAE Task Force on Classification of Status Epilepticus. Epilepsia 56: 1515-1523, 2015

21) Tanaka K, Watase K, Manabe T, et al.: Epilepsy and exacerbation of brain injury in mice lacking the glutamate transporter GLT-1. Science 276: 1699-1702, 1997

22) Sloan SA, Barres BA: The detrimental role of glial acidification during ischemia. Neuron 81: 221-223, 2014

23) Beppu K, Sasaki T, Tanaka KF, et al.: Optogenetic countering of glial acidosis suppresses glial glutamate release and ischemic brain damage. Neuron 81: 314-320, 2014

24) Szydlowska K, Tymianski M: Calcium, ischemia and excitotoxicity. Cell Calcium 47: 122-129, 2010

25) Rogawski MA: Revisiting AMPA receptors as an antiepileptic drug target. Epilepsy Curr 11: 56-63, 2011

26) Plosker GL: Perampanel: as adjunctive therapy in patients with partial-onset seizures. CNS Drugs 26: 1085-1096, 2012

27) Niu HX, Wang JZ, Wang DL, et al.: The orally active noncompetitive AMPAR antagonist perampanel attenuates focal cerebral ischemia injury in rats. Cell Mol Neurobiol 38: 459-466, 2018

28) Nakajima M, Suda S, Sowa K, et al.: AMPA receptor antagonist perampanel ameliorates post-stroke functional and cognitive impairments. Neuroscience 386: 256-264, 2018

29) Chonan M, Saito R, Kanamori M, et al.: Experience of low dose perampanel to add-on in glioma patients with levetiracetam-uncontrollable epilepsy. Neurol Med Chir (Tokyo) 60: 37-44, 2020

30) Parsons MW, Li T, Barber PA, et al.: Combined (1)H MR spectroscopy and diffusion-weighted MRI improves the prediction of stroke outcome. Neurology 55: 498-505, 2000

31) Bartnik-Olson BL, Ding D, Howe J, Shah A, Losey T: Glutamate metabolism in temporal lobe epilepsy as revealed by dynamic proton MRS following the infusion of [U13-C] glucose. Epilepsy Res 136: 46-53, 2017
32) Peeling J, Sutherland G: $1 \mathrm{H}$ magnetic resonance spectroscopy of extracts of human epileptic neocortex and hippocampus. Neurology 43: 589-594, 1993

33) Hanefeld F, Kruse B, Holzbach U, et al.: Hemimegalencephaly: localized proton magnetic resonance spectroscopy in vivo. Epilepsia 36: 1215-1224, 1995

34) Duncan JS: Imaging and epilepsy. Brain 120: 339377, 1997

35) Claassen J, Hirsch LJ, Kreiter KT, et al.: Quantitative continuous EEG for detecting delayed cerebral ischemia in patients with poor-grade subarachnoid hemorrhage. Clin Neurophysiol 115: 2699-2710, 2004

36) Finnigan SP, Walsh M, Rose SE, Chalk JB: Quantitative EEG indices of sub-acute ischaemic stroke correlate with clinical outcomes. Clin Neurophysiol 118: 2525-2532, 2007

37) Finnigan S, Wong A, Read S: Defining abnormal slow EEG activity in acute ischaemic stroke: delta/alpha ratio as an optimal QEEG index. Clin Neurophysiol 127: 1452-1459, 2016

38) Plotnikov MB, Vaizova OE, Suslov NI: Analysis of changes in the electroencephalogram power spectrum on a new model of brain ischemia in rats. Bull Exp Biol Med 118: 1257-1259, 1994

39) Sterman MB: Power spectral analysis of EEG characteristics during sleep in epileptics. Epilepsia 22: 95-106, 1981

40) Csercsa R, Dombovári B, Fabó D, et al.: Laminar analysis of slow wave activity in humans. Brain 133: 2814-2829, 2010

41) Kim SK, Cho KO, Kim SY: The plasticity of posterior communicating artery influences on the outcome of white matter injury induced by chronic cerebral hypoperfusion in rats. Neurol Res 31: 245-250, 2009

42) Barbosa GHL, Batista SP, Dos Santos PB, Thomaz CRC, Scorza FA, Cysneiros RM: Single neonatal status epilepticus does not impair cognitive function in rats. Epilepsy Behav 72: 200-202, 2017

43) Castelhano AS, Scorza FA, Teixeira MC, Arida RM, Cavalheiro EA, Cysneiros RM: Social play impairment following status epilepticus during early development. J Neural Transm (Vienna) 117: 1155-1160, 2010

44) Leite IS, Castelhano AS, Cysneiros RM: Effect of diazepam on sociability of rats submitted to neonatal seizures. Data Brief 7: 686-691, 2016

45) Farkas E, Institóris A, Domoki F, Mihály, A, Bari F: The effect of pre- and posttreatment with diazoxide on the early phase of chronic cerebral hypoperfusion in the rat. Brain Res 1087: 168-174, 2006

Corresponding author: Nobuhiro Mikuni, MD, PhD Department of Neurosurgery, Sapporo Medical University, South1 West16, Chuo-ku, Sapporo, Hokkaido 060-8543, Japan. e-mail: mikunin@sapmed.ac.jp 